\title{
Exon sequence requirements for excision in vivo of the bacterial group II intron RmInt1
}

\author{
Antonio Barrientos-Durán ${ }^{1,2}$, Isabel Chillón , Francisco Martínez-Abarca ${ }^{1}$ and Nicolás Toro ${ }^{1 *}$
}

\begin{abstract}
Background: Group II intron splicing proceeds through two sequential transesterification reactions in which the 5' and 3'-exons are joined together and the lariat intron is released. The intron-encoded protein (IEP) assists the splicing of the intron in vivo and remains bound to the excised intron lariat RNA in a ribonucleoprotein particle (RNP) that promotes intron mobility. Exon recognition occurs through base-pairing interactions between two guide sequences on the ribozyme domain $\mathrm{dl}$ known as EBS1 and EBS2 and two stretches of sequence known as IBS1 and IBS2 on the $5^{\prime}$ exon, whereas the $3^{\prime}$ exon is recognized through interaction with the sequence immediately upstream from EBS1 [( $\delta-\delta^{\prime}$ interaction (subgroup IIA)] or with a nucleotide [(EBS3-IBS3 interaction (subgroup IIB and IIC))] located in the coordination-loop of $\mathrm{dl}$. The $\delta$ nucleotide is involved in base pairing with another intron residue $\left(\delta^{\prime}\right)$ in subgroup IIB introns and this interaction facilitates base pairing between the $5^{\prime}$ exon and the intron.

Results: In this study, we investigated nucleotide requirements in the distal 5'- and 3' exon regions, EBS-IBS interactions and $\delta$ - $\delta^{\prime}$ pairing for excision of the group IIB intron Rmlnt1 in vivo. We found that the EBS1-IBS1 interaction was required and sufficient for RmInt1 excision. In addition, we provide evidence for the occurrence of canonical $\delta$ - $\delta^{\prime}$ pairing and its importance for the intron excision in vivo.

Conclusions: The excision in vivo of the Rmlnt1 intron is a favored process, with very few constraints for sequence recognition in both the $5^{\prime}$ and $3^{\prime}$-exons. Our results contribute to understand how group $I$ introns spread in nature, and might facilitate the use of Rmlnt1 in gene targeting.
\end{abstract}

\section{Background}

Group II introns are ribozymes and mobile retroelements consisting of a highly structured RNA organized into six distinct domains (dI to dVI). They have a multifunctional intron-encoded protein (IEP) ORF in dIV, encoding a reverse transcriptase-maturase that is required in vivo for the folding of the intron RNA into a catalytically active structure [1]. Group II introns splice via a lariat intermediate, in a mechanism similar to that of spliceosomal introns, via two sequential transesterification reactions [1]. In the first step, the 2'-OH group of a branch-point nucleotide residue, usually a bulged adenosine in dVI, attacks the 5' splice junction, resulting in cleavage of the 5' exon and the formation of an intron3' exon branched lariat intermediate. The released 5'

\footnotetext{
* Correspondence: ntoro@eez.csic.es

${ }^{1}$ Grupo de Ecología Genética, Estación Experimental del Zaidín, Consejo Superior de Investigaciones Científicas (CSIC). C/Profesor Albareda 1, 18008 Granada, Spain

Full list of author information is available at the end of the article
}

exon remains associated with the intron via base pairing of the intron binding sites (IBS1 and IBS2) to the exon binding sites (EBS1 and EBS2) located in ribozyme domain $\mathrm{dI}$ [2]. In the second step, the free 3'-OH of the 5 ' exon attacks the 3' splice junction, leading to the release of the intron lariat and the ligation of the 5 ' and 3' exons. Recognition of the 3'exon involves two additional base-pair interactions: the first involves the formation of a single tertiary base-pair between the last position of the intron $\left(\gamma^{\prime}\right)$ and an intron nucleotide $(\gamma)$ between dI and dII, whereas the second involves the first positions of the exon and specific intron nucleotides. The identity of the intron-binding site in the 3'exon differs between the major classes of group II introns. For IIA introns, the sequence immediately upstream from EBS1 ( $\delta$ sequence) base pairs with one to three nucleotide residues ( $\delta$ ' position) of the 3 ' exon for intron splicing (i.e., $3 \mathrm{nt}$ in the Lactococcus lactis Ll. LtrB intron). By contrast, most IIB and IIC introns display no statistical evidence of pairing of this $\delta$ position
C Biomed Central

두 2011 Barrientos-Durán et al; licensee BioMed Central Ltd. This is an Open Access article distributed under the terms of the Creative Commons Attribution License (http://creativecommons.org/licenses/by/2.0), which permits unrestricted use, distribution, and reproduction in any medium, provided the original work is properly cited. 
with the 3' exon. These group II ribozymes recognize the first nucleotide in the 3' exon, referred to as IBS3, rather than $\delta$, by canonical base-pairing with an intron nucleotide, denoted EBS3 rather than $\delta$, located in the "coordination loop" of dI $[3,4]$. The $\delta$ position is involved in a new tertiary interaction with another residue (denoted $\delta^{\prime}$ ) also located in the coordination loop [3]. These loop nucleotides are involved in aligning the two exons for the second step of splicing $\left(\delta-\delta^{\prime}\right.$ and IBS3-EBS3 interactions).

The RmInt1 intron of Sinorhizobium meliloti, a group IIB intron, is a mobile intron that self-splices in vitro in the absence of the IEP [5,6]. Recently, we investigated RmInt1 splicing activity in vivo and observed a low efficiency; only $0.07 \%$ of exons were joined [7]. This result suggests that bacterial group II introns function more like retroelements than spliceosomal introns. Like other mobile group II introns, RmInt1 recognizes DNA target sequences through both the IEP and base pairing of the intron RNA. Group II IEPs appear to recognize sequences in both the distal 5' and 3' exons, but appear to have no critical nucleotide residues in common. RmInt1 has less stringent requirements for recognition of the distal 5' and 3' exon regions, with T-15 and G+4 the only critical nucleotide residues [8], but is sufficiently long to confer high specificity. The RmInt1 IEP may also interact with nucleotide residues -20 to -16 in the distal 5'-exon, because the elimination of these residues decreases retrohoming efficiency. Despite our knowledge of RmInt1 DNA target-site recognition requirements for reverse splicing and intron mobility, little is known about the exon sequences required for intron excision due to forward splicing.

In this study, we analyzed the nucleotide requirements in the distal 5' and 3' exon regions, EBS-IBS interactions and $\delta-\delta$ pairing for excision of the IIB intron RmInt1 in vivo. We found that exon recognition by the intron is subject to few constraints, the most critical of which is the EBS1-IBS1 interaction and the $\delta$ - $\delta$ ' pairing. Our findings might have practical implications for the development of RmInt1 as a highly efficient gene targeting vector and support the extraordinary ability of RmInt1 for its dispersal in nature.

\section{Results and Discussion}

5' exon nucleotide sequence requirements for RmInt1 excision in vivo

Initial studies with the yeast aI5 $\gamma$ group II intron showed that in vitro trans-splicing depends on exon-intron binding sequences, the EBS1-IBS1 and EBS2-IBS2 pairings, whereas normal cis-splicing requires only the EBS1-IBS1 pairings [9-11]. On the basis of these results, we analyzed the 5 ' exon nucleotide sequence requirements for RmInt1 excision in vivo, by introducing single and multiple nucleotide substitutions into the IBS1 and IBS2 elements (Figure 1) that in the DNA target site blocks intron homing [8]. The linker nucleotide separating the IBS2 and IBS1 elements and the critical position -15 in the distal 5' exon for homing, [8], which has previously been suggested to be involved in a secondary IBS2* element [5], were also analyzed.

The excision reaction was characterized by primer extension; with a primer $\mathrm{P}$ complementary to a sequence located 80-97 nt from the 5' end of the intron. In these assays, the excised intron RNA was detected as a $97 \mathrm{nt}$ extension product (Figure 2A). In addition to the excised intron lariat, the former product could potentially be generated from either linear intron or lariat-3' exon intermediates. However, we have no evidence that RmInt1 generates excised linear intron molecules in vivo: no linear intron RNA was detected by 5'RLMRACE (RNA ligase-mediated rapid amplification of cDNA 5'ends), primer extension or cRT-PCR (data not shown). Moreover, we showed previously that the lariat form accumulates in the splicing process and that this product is free of lariat-3' exon reaction intermediates Likewise, the RmInt1 splicing process was inefficient, shown by a low detection of ligated exons, more likely to be susceptible to cell degradation [7].

The IBS2 stretch encompasses 5 nt (positions -13 to -9) in the 5' exon of RmInt1 that form Watson-Crick pairs with the EBS2 intron RNA sequence (Figure 1). As shown in Figure 2, the individual replacement, within IBS2, of the U-12 nucleotide by a G residue (lane 4), the

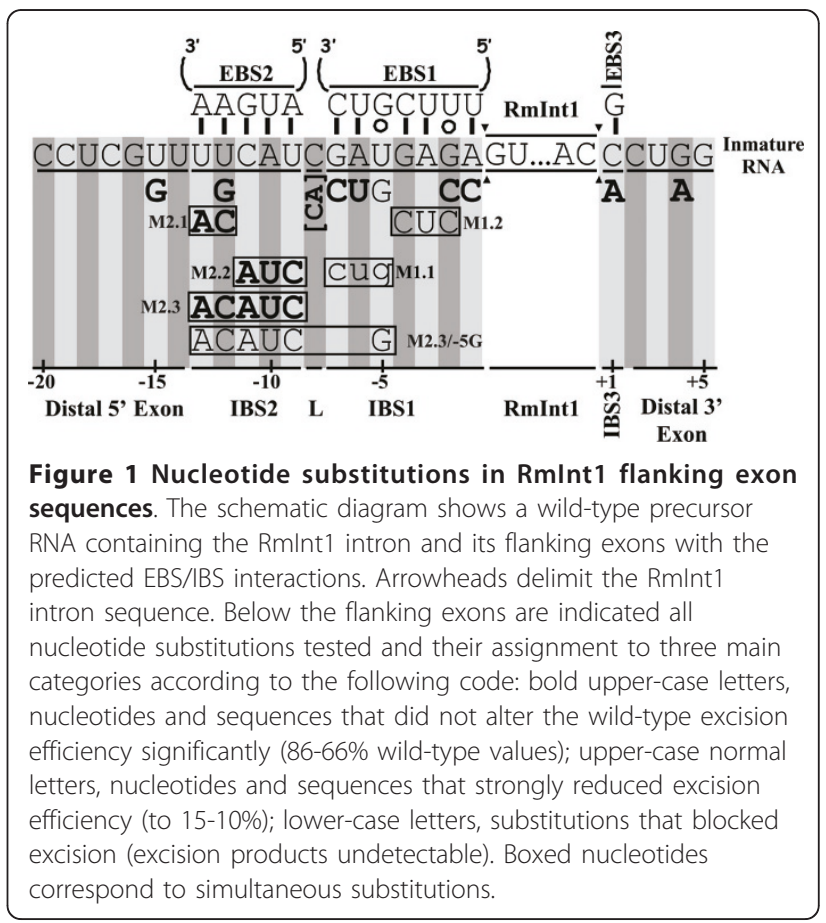




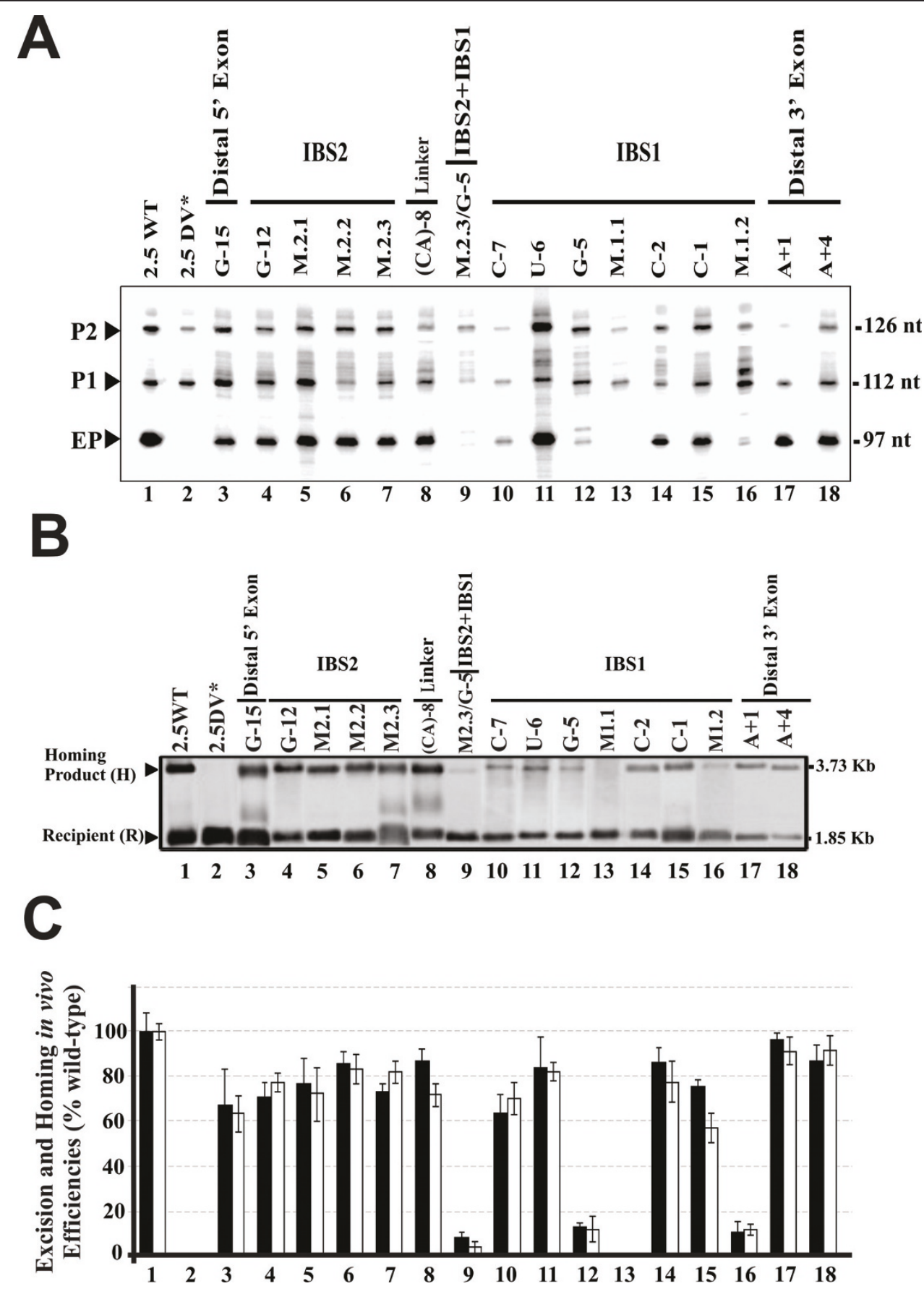

Figure 2 Effect of exon sequence mutations in intron excision and homing efficiencies. (A) Autoradiograph of the products obtained in a primer extension assay on RNPs isolated from the S. meliloti RMO17 strain harboring the wild-type intron pKG2.5 (2.5WT), the excision-defective $\mathrm{dV}$ intron mutant pKG2.5D5-CGA (2.5DV*) or the exon mutations generated for this study. The RNP preparations were reverse transcribed with the intron-specific primer, $P$. Three main products were obtained: (EP) a $97 \mathrm{nt}$ CDNA product corresponding to the excised intron RNA; and the (P1) and (P2) cDNA products (112 and $126 \mathrm{nt}$ in size, respectively) that are derived from unspliced precursor transcripts, which appear to be processed at specific $5^{\prime} U$ residues as indicated by RACE mapping in the wild-type donor construct. (B) Representative hybridization blot of homing assays. Plasmid pools from S. meliloti RMO17 harboring the wild-type pKG2.5 (2.5WT), the excision-defective dV intron mutant pKG2.5D5CGA $\left(2.5 D^{*}\right)$ or the exon mutated intron donors with the pJB0.6LAG target-recipient plasmid were analyzed by Sall digestion and Southern hybridization with an exon-specific probe. The hybridization signals and the size corresponding to the target recipient plasmid (R) and the homing product $(\mathrm{H})$ are indicated. Donor plasmid hybridization signal were removed. (C) Bar graph showing the excision (black bars) or homing (white bars) efficiencies, relative to the wild-type, into each of the intron constructs generated, calculated as indicated in Methods. Data are the means of determinations in at least four independent assays with the SD indicated by thin lines. 
combined replacements of the $\mathrm{U}-13$ and $\mathrm{U}-12$ residues with AC (mutant M2.1, lane 5), the replacement of the C-11, A-10 and U-9 nucleotides with AUC (mutant M2.2, lane 6) and complete replacement of the IBS2 sequence UUCAU with ACAUC (mutant M2.3, lane 7) in a construct (pKG2.5) in which the intron is flanked by long stretches of exon sequences $(-176 /+466)$, moderately decreased excised intron levels ( 71 to $85 \%$ of wild-type). A similar result was obtained when the IBS2 sequence was replaced in a construct in which RmInt1 was flanked by short stretches of its natural 5' (-20 nt) and 3' exon (+5 nt) sequences (not shown).

Over the distal 5' region, the replacement of U-15 with a $G$ residue resulted in the retention of $\sim 67 \%$ of wild-type intron RNA excision activity (lane 3 ). The extension of the linker nucleotide between IBS2 and IBS1 by the addition of one nucleotide (C-8 to $\mathrm{CA}$, lane 8 ) led to a moderate decrease in excision efficiency ( $87 \%$ of wild-type levels). Thus, in contrast to the critical positions of the EBS-IBS interactions in the DNA Target [8], neither the distal 5'exon region, IBS2-EBS2 nor putative IBS2*-EBS2 [5] pairings are required for intron excision.

By contrast, more heterogeneous results were obtained for nucleotide substitutions within the IBS1 element. The IBS1 stretch encompasses $7 \mathrm{nt}$, positions -7 to -1 with respect for the intron insertion site, that base-pair with the EBS1 sequence in the intron RNA (Figure 1). Single substitutions at the 5 ' end, such as the replacement of G-7 with a $\mathrm{C}$ residue and of A-6 with a $U$ residue, resulted in excision efficiencies of 63 and $86 \%$ those of the wild-type, respectively (Figure $2 \mathrm{~A}$ and $2 \mathrm{~B}$, lanes 10 and 11). Similar results were obtained for individual nucleotide substitutions at the 3' end of the IBS1 sequence: $\mathrm{G}-2$ to $\mathrm{C}(86.5 \%$, lane 14$)$ and $\mathrm{A}-1$ to $\mathrm{C}$ (75.2\%, lane 15). By contrast, the replacement of the U5 nucleotide with a $\mathrm{G}$ residue strongly reduced excision to $12 \%$ wild-type levels (lane 12 ), suggesting that this is a critical nucleotide position for cleavage, presumably at the 5' splice site.

Simultaneous changes were also made within the IBS1 element. We focused on simultaneous substitutions in positions $-7,-6$ and -5 (mutant M1.1, GAU to CUG) that rendered excision undetectable, and simultaneous changes of the $-4,-3$ and -2 nucleotides (GAG to CUC, mutant M1.2), which strongly reduced intron excision ( $13 \%$ of wild-type levels). These results identified the IBS1 element in the 5' exon as the most critical for the intron excision reaction. A combined mutant (M2.3/-5G) lacking the canonical IBS2 sequence plus the substitution of the U-5 nucleotides by a G residue in IBS1 rendered intron excision barely detectable (lane 9), which reveals the additional contribution of the IBS2-EBS2 pairing to the efficiency of the intron excision reaction.

\section{3' exon nucleotide sequence requirements for RmInt 1 excision in vivo}

In class IIB introns, position +1 at the 3 ' exon, known as IBS3 ( $\mathrm{C}+1$ residue in the RmInt1 3' exon), base-pairs with the EBS3 element in the intron RNA (position 329G), which is located in the "coordination loop" at dI and displaced only one nucleotide from the 3 ' branch of helix ID ${ }^{\text {(iv) }}[3]$. The IBS3-EBS3 interaction has been shown to be important for the second step of splicing [3] and for RmInt1 target-site recognition for intron mobility [8]. Interestingly, the RmInt1 donor construct in which IBS3 $\mathrm{C}+1$ was replaced by an A residue displayed no significant impairment of excision activity ( $~ 96 \%$ of wild-type activity, Figure $2 \mathrm{~A}$ and $2 \mathrm{~B}$, lane 17 ). In addition, replacement of the $\mathrm{G}+4$ residue in the 3' exon with an A residue, which has a $40 \%$ of reduction on homing efficiency when the target is mutated [8], resulted in an excision efficiency $87 \%$ that of the wild type (lane 18). One possible interpretation of our data is that the substitution of IBS3 $\mathrm{C}+1$ by an A residue blocks second step of splicing and the $97 \mathrm{nt}$ extension product is generated from lariat-3'exon intermediates. To solve this question, we performed qRT-PCR and measured exon ligation by the $A+1$ mutant. As shown in Table 1 the splicing activity of the former mutant was equivalent to that by the wild-type intron. We therefore conclude that neither IBS3-EBS3 pairing nor 3' exon sequences are required for RmInt1 excision in vivo.

\section{The excision process is a limiting step for RmInt 1 intron mobility}

The above results suggest that the RmInt1 excision in vivo is a highly favored process, with IBS1-EBS1 pairing the most critical step. As the intron lariat released from the 3'exon remains associated with the IEP, forming a ribonucleoprotein particle complex (RNP) that mediates intron mobility, we investigated whether the observed decrease in excision was correlated with reduced intron mobility. We carried out a homing assay with intron donor and recipient plasmids, in which intron mobility was assessed by DNA hybridization. Once RmInt1 was excised in vivo from the exon-mutant donor constructs, the RNPs produced were wild-type. Following introduction of either the wild-type intron (pKG2.5) or mutant constructs into a S. meliloti RMO17 strain harboring

\begin{tabular}{cc}
$\begin{array}{l}\text { Table } 1 \mathbf{~ R m I n t} 1 \text { splicing activity in S.meliloti measured by } \\
\text { relative quantification of joined exons in qRT-PCR }\end{array}$ \\
\hline Construction & $\%$ splicing \pm error $^{\text {a }}$ \\
\hline WT & 100 \\
DV & $0.76 \pm 0.01$ \\
$+1 \mathrm{~A}$ & $98 \pm 1$ \\
\hline${ }^{*}$ Rmlnt1 splicing is calculated by $2^{-\Delta \Delta C}$ Method (21) using qRT-PCR primers
\end{tabular}

${ }^{a}$ Rmlnt1 splicing is calculated by $2^{-\Delta \Delta C}$ Method (21) using qRT-PCR primers and conditions specified in Methods section. 
the target-recipient plasmid pJB0.6as, a broad correspondence was observed between the excision level detected by primer extension and homing efficiency (compare black and white bars in Figure 2C).

\section{Requirement for $\delta$ - $\boldsymbol{\delta}^{\prime}$ interactions in the excision in vivo of} the RmInt1 intron

The $\delta-\delta$ ' interaction is thought to be required for the splicing reaction and seems to facilitate base pairing between the 5 'exon and the intron; disruption of this interaction interferes with exon binding [3]. In the case of RmInt1, this interaction involves residues 266G $(\delta)$ and $227 \mathrm{C}\left(\delta^{\prime}\right)$ (Figure $\left.3 \mathrm{~A}\right)$. We found that the disruption of this pairing in the RmInt1 intron (Figure 3B), through the replacement of the $\delta$ residue G266 nucleotide with a $C$ residue $(\delta C)$ strongly decreased excision (to $\sim 8 \%$ of wild-type levels), whereas the introduction of a compensatory $\delta^{\prime} \mathrm{C}$ to $\mathrm{G}$ mutation $(\delta-\delta$ ', C-G instead of the wildtype $\mathrm{G}-\mathrm{C}$ pairing) restored excision to the level of the wild-type intron (lanes 2 and 3, respectively). Consistent with the lower excision efficiency observed following disruption of the $\delta-\delta$ ' interaction ( $\delta \mathrm{C}$ mutant), mutant mobility was undetectable at both the wild-type DNA target site and a mutant target site at which the IBS3 nucleotide $+1 \mathrm{C}$ was replaced with a $+1 \mathrm{G}$ nucleotide (Figure 3C, lanes 3 and 4). As expected, the introduction
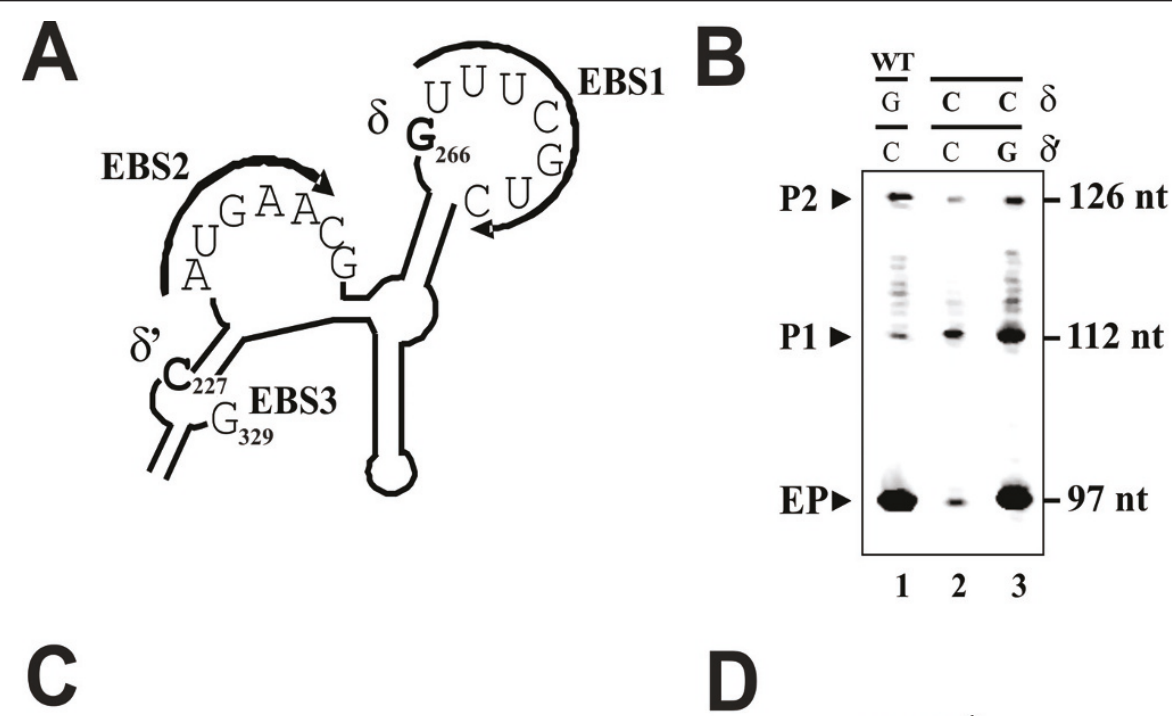

\section{DONOR OF RmInt1 AND DERIVATIVES pKG2.5 (7.9 kb)}
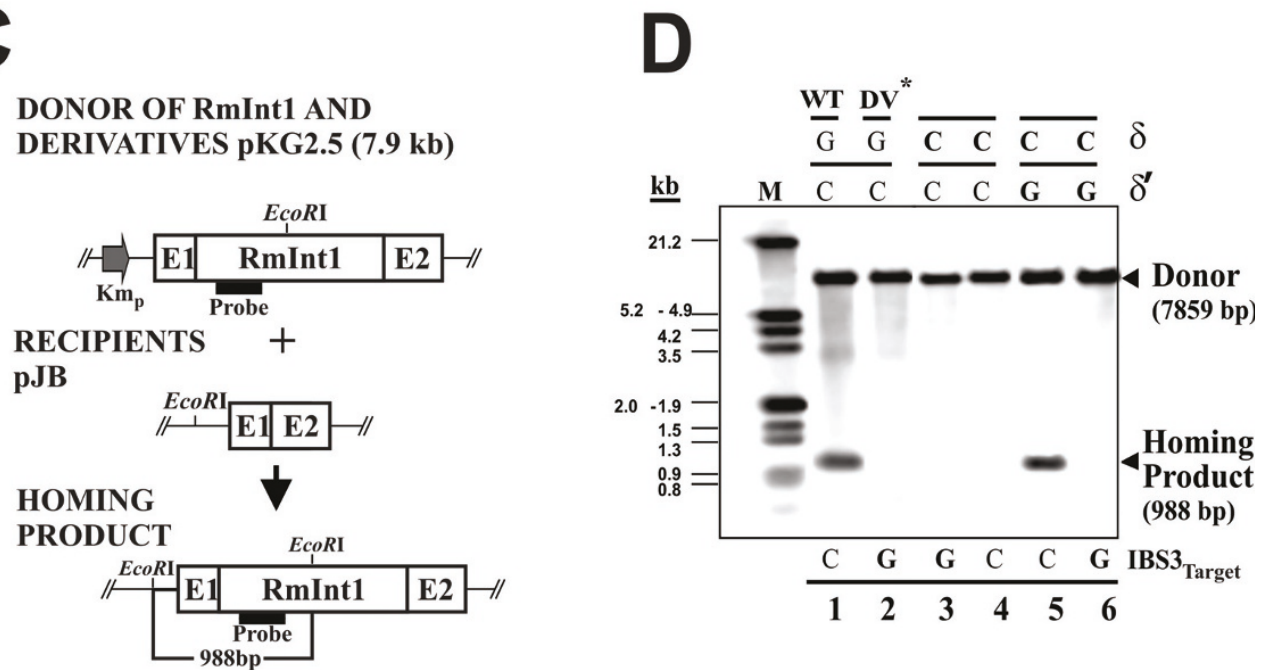

Figure 3 Study of the role of the $\boldsymbol{\delta}-\boldsymbol{\delta}^{\prime}$ interaction in RmInt1 excision in vivo. (A) Schematic diagram of domain dl focusing on the region containing the EBSs $(1,2$ and 3$), \delta$ and $\delta^{\prime}$ elements, which are shown in bold typeface. (B) Autoradiograph of the products generated in the primer extension assay. Modifications to the $\delta$ and $\delta^{\prime}$ wild-type elements in the different mutants are indicated in bold typeface. (C) Diagram of the double plasmid homing assay performed showing Intron donor, recipient plasmids containing Rmlnt1 target sites (E1+E2), and homing products. Relevant restriction sites and the sizes of DNA fragments used as diagnostic of homing are indicated (D) Southern blot obtained in homing assays between derivative donor plasmids (pKG2.5 with modifications of the aforementioned elements) and the wild-type recipient plasmid pJB-20/+5 containing the homing site or a mutant (pJB-20/+5; IBS3 G) shown in bold typeface. An Rmlnt1 specific probe was used, after the EcoRl digestion of plasmid DNA pools for the detection of donor and homing products. 
into the $\delta \mathrm{C}$ mutant of the compensatory $\delta^{\prime} \mathrm{C}$ to $\mathrm{G}$ mutation to restore $\delta-\delta$ ' pairing, (C-G instead of the wildtype G-C pairing) re-established mobility at the wildtype target (lane 5), but not at the mutant target carrying a $\mathrm{G}$ nucleotide at position +1 (lane 6). These results provide evidence for the occurrence of intradomain dI canonical $\delta$ - $\delta$ ' pairing in vivo and its importance for RmInt1 excision.

\section{Conclusions}

We show here that the intron-exon interaction IBS1EBS1 and $\delta-\delta$ 'interaction in the domain dI of the rybozime, play an important role in the RmInt1 excision in vivo. By contrast, however, IBS2, residue $\mathrm{U}-15$ in the distal 5' exon and IBS3 or G+4 in the 3' exon are not required for the excision reaction, despite the requirement of these elements for the homing process. Nucleotide replacements causing a moderate or severe decrease in the excision efficiency of the intron displayed a similar decrease in homing efficiencies, probably due to a decrease in the production of RNPs. Thus, RmInt1 has different exon nucleotide sequence requirements in either both forward (intron excision) and reverse (intron retrohoming) splicing reactions. Our results contribute to understand how group II introns spread in nature, and might facilitate the use of RmInt1 in gene targeting.

Group II introns can move site-specifically to homologous intronless genes, in a process known as retrohoming or, at much lower frequencies, to novel ectopic sites, in a process known as retrotransposition. It is believed that the latter process has led to the wide dispersal of mobile group II introns among bacterial species and also may have been used to invade eukaryotic genomes, where mobile group II intron are thought to have evolved into spliceosomal introns and non-long-terminal-repeat retrotransposons [12]. Intron insertion-sites with good EBS/ IBS pairings suggest insertion via retrohoming, whereas poorly matched IBS sequences suggest insertion via retrotransposition. It is generally accepted that only those introns inserted by retrohoming at sites with complementary IBS sequences are expected to splice efficiently. However, we show here that RmInt1 excision in vivo is subject to few constraints, the most critical of which is the EBS1-IBS1 interaction. Thus, the spread of group II introns into bacterial genomes from ectopic sites to homing sites may occur efficiently, increasing their ability to spread in nature.

Gene targeting technology based on group II introns requires the modification of IBS2, IBS1 and IBS3, to render these sites complementary to the retargeted EBS2, EBS1 and EBS3 sequences for efficient RNA splicing $[13,14]$. We have recently demonstrated that the RmInt1 intron could be used for gene targeting, following the design of introns for insertion into different target sites [15]. As the predicted base-pairing interactions with the RmInt1 intron RNA - EBS2-IBS2 and EBS3-IBS3 - have only a small effect on overall intronsplicing efficiency, we hypothesize that the provision of IBS2 and IBS3 nucleotide combinations complementary to modified intron EBS2 and EBS3 sequences in intron plasmid constructs may not be necessary, potentially facilitating the use of RmInt1 in gene targeting.

\section{Methods}

\section{Bacterial strains, media and growth conditions}

Rhizobial strain S. meliloti RMO17, lacking the RmInt1 intron, was grown at $28^{\circ} \mathrm{C}$ on TY or defined minimal medium [16]. E. coli DH5 $\alpha$ was used for cloning and the maintenance of recombinant plasmids; it was cultured at $37^{\circ} \mathrm{C}$ in Luria-Bertani medium [17]. Antibiotics were added to the medium when required, at the following concentrations: kanamycin, $200 \mu \mathrm{g} / \mathrm{ml}$ for S. meliloti and $50 \mu \mathrm{g} / \mathrm{ml}$ for E. coli; ampicillin, $200 \mu \mathrm{g} / \mathrm{ml}$ and tetracycline, $10 \mu \mathrm{g} / \mathrm{ml}$.

\section{RmInt 1 and its intron derivatives}

RmInt1 donor derivatives containing point or accumulative substitutions in the flanking regions of intron insertion sites and ribozyme domain dI, including the $\delta$ and $\delta$ ' sequences, were generated with the Altered Sites II in vitro Mutagenesis System (Promega, Madison, WI), using PAL2.5 as a template. This plasmid was generated by inserting a $2.5 \mathrm{~kb} S p h \mathrm{I}$ fragment containing the entire RmInt1 sequence and flanking exons $(-174 /+466)$ from pGEmex2.5 [18] into the pALTER-1 vector. Nucleotide substitutions were checked by sequencing and the mutated $2.5 \mathrm{~kb}$ DNA fragment was retrieved from pAL2.5 as a BamHI/SpeI fragment and inserted into pKG0 (pKG2.5 and mutant derivatives), which constitutively expresses RmInt1 and its derivatives under control of the promoter of the $\mathrm{Km}$ resistance gene [18].

\section{RNP isolation}

RNPs were isolated essentially as previously described $[19,20]$. RNPs were isolated by picking a single colony from a plate of each bacterial strain and using it to inoculate $3 \mathrm{ml}$ of TY medium, which was then incubated at $28^{\circ} \mathrm{C}$ for two days, allowing the cells to reach stationary phase. We then added $1 \mathrm{ml}$ of the culture to $200 \mathrm{ml}$ of TY medium in a $500 \mathrm{ml}$ flask and incubated the flask at $28^{\circ} \mathrm{C}$ until the culture reached the exponential growth phase $\left(\mathrm{OD}_{600 \mathrm{~nm}}\right.$ of 0.6 to 0.9$)$.

\section{Primer extension assays}

These experiments were carried out on RNP preparations, since the lariat intron seems to be less susceptible to degradation than ligated exons, due to its association with the IEP forming ribonucleoprotein particles 
(RNPs). Primer extension reactions were essentially carried out as previously described [20]. Samples were then resolved by electrophoresis in a denaturing $6 \%$ polyacrylamide gel. The cDNA bands corresponding to the resolved extension products were quantified with the Quantity One software package (Bio-Rad Laboratories). We assume that the generated mutations in the exons do not affect intron RNA stability, which is mainly affected by its interaction with the IEP. Thus, the excision efficiency was calculated as the ratio of the EP (97 nt) product from each intron construct to wild-type construct, normalized with respect to the sum of P1 and P2 (112 and $126 \mathrm{nt}$ ) unspliced precursor products and expressed as a percentage.

\section{qRT-PCR}

For the quantification of ligated RmInt1 flanking exons, a 283 bp fragment was amplified with 3'RTSp (5'-ATTCAACGGGAAACGTCTTG) and +9 (5'-AGCGGCGCCATGTTCGTCTT) primers. To normalize the loading amount of the starting material, a $500 \mathrm{bp}$ fragment of the $16 \mathrm{~S}$ ribosomal subunit RNA was amplified with $S$. meliloti specific primers Sino 452 (5'-CGTTGTTCG GAATTACTGG) and Sino 952 (5'-TGTCTCCGATCCAGC). Primer specificity was determined by meltingcurve analysis and gel electrophoresis (Additional File 1 ). We conducted the qRT-PCR analysis by using the iCycler System (Bio-Rad, Additional File 2). Each reaction was run in triplicates and contained $5 \mu \mathrm{l}$ of cDNA template (equivalent to $500 \mathrm{ng}$ total RNA), $0.5 \mathrm{mM}$ dNTP, 5 pmol of each oligonucleotide primer, $7.5 \mathrm{mM}$ $\mathrm{MgCl}_{2}, 2.5 \mu \mathrm{l}$ of a 1:10,000 dilution of SYBR Green, 2.3 $\mu \mathrm{l}$ of reaction buffer and $0.5 \mathrm{U}$ of Platinum Taq DNA Polymerase (Invitrogen), with the final volume made up to $23 \mu \mathrm{l}$ with DEPC water. The PCR cycling conditions were as follows: hot start, with heating at $94^{\circ} \mathrm{C}$ for 3 min, followed by 40 cycles of denaturation at $94^{\circ} \mathrm{C}$ for $30 \mathrm{~s}$, annealing at $55^{\circ} \mathrm{C}$ for $30 \mathrm{~s}$, and extension at $72^{\circ} \mathrm{C}$ for $30 \mathrm{~s}$. Controls without a template were also included. Two independent RNA preparations from each strain were tested. Relative quantification was carried out for splicing, based on the $2^{-\Delta \Delta C T}$ method [21]. Briefly, RmInt1 splicing is defined by the following equation: $2^{-\Delta \Delta C T}$ where $\Delta \Delta C T=\Delta C T$ ( $\mathrm{DV}^{*}$ or $+1 \mathrm{~A}$ ) $\Delta \mathrm{CT}(\mathrm{WT}) . \Delta \mathrm{CT}\left(\mathrm{DV}^{*}\right.$ or $\left.+1 \mathrm{~A}\right)=\mathrm{CT}(\mathrm{DV}$ or $+1 \mathrm{~A}$ mRNA amplified by 5-6 primers) - CT (DV or $+1 \mathrm{~A}$ mRNA amplified by $S$. meliloti specific $16 \mathrm{~S}$ primers Sino 452 and Sino952). $\triangle \mathrm{CT}(\mathrm{WT})=\mathrm{CT}$ (WT mRNA amplified by 5-6 primers) - CT (WT mRNA amplified by $S$. meliloti specific $16 \mathrm{~S}$ rRNA primers Sino 452 and Sino952). The result is expressed as a percentage related to WT construction. The error is calculated applying the formula: $2^{-\Delta \Delta C T} \cdot \ln 2 \cdot \Sigma S E$.

\section{Homing assays}

Double-plasmid homing assays were performed using the S. meliloti RmInt1-less strain RMO17. We studied homing between compatible intron donor (pKG2.5 and mutant derivatives) and target-site recipient plasmids (pJB0.6as $(-174 /+466)$ or $\mathrm{pJB}-20 /+5)$ as previously described [22]. Plasmids were mobilized from E. coli DH5 $\alpha$ to rhizobial strains by tri-parental mating, using pRK2013 as a helper plasmid [23]. Plasmid pools were isolated from exponentially growing cultures in TY of four to ten transconjugants digested with the indicated restriction enzymes and resolved by electrophoresis in $0.8 \%$ Tris-acetate agarose gels and vacuum blotted onto nylon filters according to the manufacturer's indications (Roche Diagnostics $\mathrm{GmbH}$, Mannheim, Germany). DNA hybridization was performed under high-stringency conditions, with DIGlabeled probes generated by PCR and specific for the ISRm2011-2 element (spanning positions 562-1025; used in Figure 2) and the RmInt1 group II intron ribozyme (spanning positions 147-594; used in Figure 3). Hybridizing bands corresponding to homing products $(\mathrm{H})$ and recipient plasmids $(\mathrm{R})$ were analyzed with Quantity One software (Bio-Rad Laboratories) and invasion efficiency of each target site calculated as $100[\mathrm{H} /(\mathrm{H}+\mathrm{R})][18]$.

\section{Additional material}

Additional file 1: Specificity of primer pairs used in real-time PCR. A 40-cycle end-point PCR reaction was carried out with primers 3'RTSp and +9 to amplify ligated Rmlnt1 flanking exons and with primers Sino 452 and Sino 952 for amplifying the 165 ribosomal subunit as a normalizer of the amount of RNA.

Additional file 2: Amplification plot. Fluorescence was plotted as a function of the PCR cycle number using iQ5 software package (Bio-Rad).

\section{Acknowledgements and funding}

This work was supported by research grants BIO2008-00740 from the Spanish Ministerio de Ciencia e Innovación, CSD 2009-0006 of Programme Consolider-Ingenio 2010 and CVI-01522 from the Junta de Andalucía including ERDF (European Regional Development Fund).

\section{Author details}

${ }^{1}$ Grupo de Ecología Genética, Estación Experimental del Zaidín, Consejo Superior de Investigaciones Científicas (CSIC). C/Profesor Albareda 1, 18008 Granada, Spain. ${ }^{2}$ Department of Biochemistry, University of California, 900 University Avenue 92521, Riverside (CA) USA.

\section{Authors' contributions}

AB-D carried out the molecular genetic studies and drafted the manuscript. IC carried out the qRT-PCR assays. FMA and NT conceived and participated in the design of the study. In addition, NT coordinated the study and supervised the drafting of the manuscript. All authors have read and approved the final manuscript.

Received: 7 December 2010 Accepted: 23 May 2011 Published: 23 May 2011 


\section{References}

1. Michel F, Ferat JL: Structure and activities of group II introns. Annu Rev Biochem 1995, 64:435-461.

2. Jacquier A, Michel F: Multiple exon-binding sites in class II self-splicing introns. Cell 1987, 50:17-29.

3. Costa M, Michel F, Westhof E: A three-dimensional perspective on exon binding by a group II self-splicing intron. EMBO J 2000, 19:5007-5018.

4. de Lencastre A, Hamill S, Pyle AM: A single active-site region for a group II intron. Nat Struct \& Mol Biol 2005, 12(7):626-627.

5. Costa M, Michel F, Toro N: Potential for alternative intron-exon pairings in a group II intron RmInt1 from S. meliloti and its relatives. RNA 2006, 12:338-341.

6. Costa M, Michel F, Molina-Sánchez MD, Martínez-Abarca F, Toro N: An alternative intron-exon pairings scheme implied by unexpected in vitro activities of group II intron from Sinorhizobium meliloti. Biochimie 2006, 88:711-717.

7. Chillón I, Martínez-Abarca F, Toro N: Splicing of the Sinorhizobium meliloti RmInt1 group II intron provides evidence of retroelement behavior. Nucleic Acids Res 2011, 39:1095-1104.

8. Jiménez-Zurdo Jl, García-Rodríguez FM, Barrientos-Durán A, Toro N: DNAtarget requirements for homing in vivo of a bacterial group II intron encoding a protein lacking the DNA endonuclease domain. J Mol Biol 2003, 326:413-423.

9. Michel $F$, Jacquier $A$ : Long-range intron-exon and intron-intron pairings involved in self-splicing of class II catalytic introns. Cold Spring Harbor Symposia on Quantitative Biology 1987, 52:201-212.

10. Xiang Q, Qin PZ, Michels WJ, Freeland K, Pyle AM: Sequence specificity of a group II intron ribozyme: multiple mechanisms for promoting unusually high discrimination against mismatched targets. Biochemistry 1998, 37:3839-3849.

11. Su LJ, Qin PZ, Michels WJ, Pyle AM: Guiding ribozyme cleavage through motif recognition: the mechanism of cleavage site selection by a group II intron ribozyme. J Mol Biol 2001, 306:655-668.

12. Pyle AM, Lambowitz AM: Group II introns: ribozymes that splice RNA and invade DNA. In The RNA world.. 3 edition. Edited by: Gesteland RF, Cech TR, Atkins JF. New York: Cold Spring Harbor Laboratory Press; 469-505.

13. Guo H, Karberg K, Long M, Jones JP III, Sullenger B, Lambowitz AM: Group II introns designed to insert into therapeutically relevant DNA target sites in human cells. Science 2000, 289:452-457.

14. Sambrook J, Fritsch EF, Maniatis T: Molecular cloning: a laboratory manual. Cold Spring Harbor Laboratory Press 1989.

15. García-Rodríguez FM, Barrientos-Durán A, Díaz-Prado V, Fernandéz-Ló M, Toro N: Use of Rmln1, a group IIB intron lacking the intron-encoded protein endonuclease domain, in gene targeting. Appl Environ Microbio 2011, 77:854-861.

16. Robertsen BK, Aiman P, Darvill AG, McNeil M, Alberstein P: The structure of acidic extracellular polysaccharides secreted by Rhizobium leguminosarum and Rhizobium trifolii. Plant Physiol 1981, 67:389-400.

17. Sambrook J, Fritsch EF, Maniatis T: Molecular cloning: a laboratory manual. Cold Spring Harbor Laboratory Press 1989.

18. Martínez-Abarca F, García-Rodríguez FM, Toro N: Homing of a bacterial group II intron with an intron-encoded protein lacking a recognizable endonuclease domain. Mol Microbiol 2000, 35:1405-1412.

19. Matsuura M, Saldanha R, Ma H, Wank H, Yang J, Mohr G, Cavanagh S, Dunny GM, Belfort M, Lambowitz AM: A bacterial group II intron encoding reverse transcriptase, maturase, and DNA endonuclease activities: biochemical demonstration of maturase activity and insertion of new genetic information within the intron. Genes Dev 1997, 11:2910-2924.

20. Muñoz-Adelantado E, San Filippo J, Martínez-Abarca F, García-Rodríguez FM, Lambowitz AM, Toro N: Mobility of the Sinorhizobium meliloti group II intron RmInt1 occurs by reverse splicing into DNA, but requires an unknown transcriptase priming mechanism. J Mol Biol 2003, 327:931-943.

21. Livak KJ, Schmittgen TD: Analysis of relative gene expression data using real-time quantitative PCR and the $2^{-\Delta \Delta C}$ T Method. Methods 2001, 25:402-408.

22. Martínez-Abarca F, Barrientos-Durán A, Fernández-López M, Toro N: The Rmlnt1 group II intron has two different retrohoming pathways for mobility using predominantly the nascent lagging strand at DNA replication forks for priming. NuCl Acids Res 2004, 32:2880-2888.
23. Ditta G, Stanfield S, Corbin D, Helinski DR: Broad host range DNA cloning system for Gram-negative bacteria. Construction of a gene bank of Rhizobium meliloti. Proc Natl Acad Sci USA 1980, 77:7347-7351.

doi:10.1186/1471-2199-12-24

Cite this article as: Barrientos-Durán et al:: Exon sequence requirements for excision in vivo of the bacterial group II intron Rmlnt1. BMC

Molecular Biology 2011 12:24.

\section{Submit your next manuscript to BioMed Central and take full advantage of:}

- Convenient online submission

- Thorough peer review

- No space constraints or color figure charges

- Immediate publication on acceptance

- Inclusion in PubMed, CAS, Scopus and Google Scholar

- Research which is freely available for redistribution

Submit your manuscript at www.biomedcentral.com/submit

Ciomed Central 